\title{
DERIVED LENGTH FOR ARBITRARY TOPOLOGICAL SPACES
}

\author{
A.J. JAYANTHAN \\ School of Mathematics and Computer/Information Sciences \\ University of Hyderabad \\ Central University P.O. Hyderabad 500134 \\ India \\ (Received February 8, 1988 and in revised form February 15, 1989)
}

\begin{abstract}
The notion of derived length is as old as that of ordinal numbers itself. It is also known as the Cantor-Bendixon length. It is defined only for dispersed (that is scattered) spaces. In this paper this notion has been extended in a natural way for all topological spaces such that all its pleasing properties are retained. In this process we solve a problem posed by V. Kannan. ([1] Page 158)
\end{abstract}

KEY WORDS AND PHRASES: Ordinal invariants, dispersed spaces, and derived length. 1991 AMS SUBJECT CLASSIFICATION CODES. Primary 54 C05 Secondary 54 A25, 54 G99.

\section{PRELIMINARIES.}

Suppose $(X, \tau)$ is a topological space. We denote the set of all limit points of a subset $A$ of $X$ by $A^{1}$. The space $X$ is denoted by $X^{0}$. As induction hypothesis, we assume that $X^{\beta}$ is defined for all $0 \leq \beta<\alpha$. Now we define $X^{\alpha}$ as follows.

$$
X^{\alpha}= \begin{cases}\bigcap_{\beta<\alpha} X^{\beta} & \text { if } \alpha \text { is a limit ordinal } \\ \left(X^{\beta}\right)^{1} & \text { if } \alpha=\beta+1 .\end{cases}
$$

So we obtained a chain $X=X^{0} \supset X^{1} \supset \ldots X^{\alpha} \supset X^{\alpha+1} \supset \ldots$. By standard arguments on the cardinality of the space $X$, one can show that there exists an ordinal number $\alpha$ such that $X^{\alpha}=X^{\alpha+1}=\ldots$.

PROPOSITION 0.1: For any topological space $X$, the following are equivalent:

(i) There exists an ordinal number $\alpha$ such that $X^{\alpha}$ is empty.

(ii) Every nonempty closed subspace of $X$ contains an isolated point.

(iii) Every nonempty subspace of $X$ contains an isolated point.

(iv) $X$ does not contain a dense-in-itself subspace.

PROOF. Omitted. (See [1] and [2])

DEFINITION 0.2: A topological space $X$ satisfying any one of the above equivalent conditions is called a dispersed (or scattered) space. From statement (i) of Proposition 0.1, we observe that for any dispersed space $X$, there exists an ordinal number $\alpha$ such that $X^{\alpha}$ is empty. We define the derived length of a (dispersed) space $X$ as $\inf \left\{\alpha: X^{\alpha}=\varnothing\right\}$ and denote it by $d(X)$ or $d(\tau)$ where $\tau$ is the corresponding topology considered on $X$. $[3,4]$.

First we observe some properties of derived length. 
THEOREM 0.3: For a dispersed space $X, d(X)$ satisfied the following properties.

(i) For a subspace $Y$ of $X, d(Y) \leq d(X)$.

(ii) $d(X)$ is smaller for finer topologies.

(iii) $d(Y) \leq d(X)$ if $Y$ is a continuous open image of $X$.

(iv) If $X$ is a compact space and $Y$ is a $T_{1}$-space such that $Y$ is a closed continuous image of $X$, then $d(Y) \leq d(X)$.

(v) If $X$ is the topological sum of a class $\left\{X_{i}: i \in I\right\}$ of dispersed topological spaces, then $X$ is dispersed and $d(X)=\sup \left\{d\left(X_{i}\right): i \in I\right\}$.

REMARK: A proof of this theorem can be found in [1]. This is also a consequence of later results of this paper.

\section{1. $d(X)$ FOR ALL SPACES.}

Suppose $(X, \tau)$ is an arbitrary topological space and $A$ is a subspace of $X$. A chain, $A=A_{0} \subset A_{1} \subset \ldots \subset A_{\alpha+1} \subset \ldots$ of subsets of $X$ is called a $K$-chain on $X$ with base set $A$ if for all $\alpha, A_{\alpha+1} \backslash A_{\alpha}$ is contained in $\overline{A_{\alpha} \backslash A_{\beta}}$ for all $\beta<\alpha$. By standard arguments on cardinality of $X$, we can prove that there exists an ordinal number $\alpha$ such that $A_{\alpha}=A_{\alpha+1}$. We call inf $\left\{\alpha: A_{\alpha}=A_{\alpha+1}\right\}$ the length of the chain. For notational convenience, hereafter we denote any $K$-chain $A_{0} \subset A_{1} \subset \ldots \subset A \subset A_{\alpha+1} \subset \ldots$ or simply by $\left\{A_{\alpha}\right\}$ without mentioning the indexing set or base set explicitly. The length of the chain $\left\{A_{\alpha}\right\}$ is denoted by $l\left(\left\{A_{\alpha}\right\}\right)$. We define the length of a topological space $X$ as $\sup \left\{l\left(\left\{A_{\alpha}\right\}\right):\left\{A_{\alpha}\right\}\right.$ is a $K$-chain in $\left.X\right\}$ and denote it as $l(X) . \quad l(X)$ can be verified to be a topological invariant. Now, we first note that $l$ satisfies all those properties of derived length given in Theorem 0.3.

THEOREM 1.1: $l(X)$ satisfies all five properties (i) to (v) of Theorem 0.3.

PROOF. Since the proofs of statements (i), (ii) and (v) are routine, we omit them. We prove statements (iii) and (iv) here.

(iii) Suppose $f$ is an open continuous map from $X$ onto $Y$. To prove $l(Y) \leq l(X)$, we consider an arbitrary $K$-chain $\left\{B_{\alpha}\right\}$ in $Y$. Define $A_{\alpha}=f^{-1}\left(B_{\alpha}\right)$.

CLAIM: $\left\{A_{\alpha}\right\}$ is also a $K$-chain and $l\left(\left\{A_{\alpha}\right\}\right)=l\left(\left\{B_{\alpha}\right\}\right)$.

For, suppose $x$ is a point in $A_{\alpha+1} \backslash A_{\alpha}$ and $U_{x}$ is an open neighborhood of $x$. Then, $f\left(U_{x}\right)$ is an open neighborhood of $f(x) \in B_{\alpha+1} \backslash B_{\alpha}$. Since $\left\{B_{\alpha}\right\}$ is a $K$-chain, $f\left(U_{x}\right)$ meets $B_{\alpha} \backslash B_{\beta}$ for all $\beta<\alpha$. Hence $U_{x}$ meets $A_{\alpha} \backslash A_{\beta}$ for all $\beta<\alpha$. Thus, we have proved that $\left\{A_{\alpha}\right\}$ is a $K$-chain. Also it is obvious that $A_{\alpha+1} \backslash A_{\alpha}$ is nonempty for all $\alpha$ such that $B_{\alpha+1} \backslash B_{\alpha}$ is nonempty. This establishes the claim that $l\left(\left\{A_{\alpha}\right\}\right)=l\left(\left\{B_{\alpha}\right\}\right)$. So we have $l(Y)=\sup \left\{l\left(\left\{B_{\alpha}\right\}\right):\left\{B_{\alpha}\right\}\right.$ is a $K$-chain in $\left.Y\right\}=\sup \left\{l\left(\left\{f^{-1}\left(B_{\alpha}\right)\right\}:\left\{B_{\alpha}\right\}\right.\right.$ is a $K$-chain in $\left.Y\right\} \leq \sup \left\{l\left(\left\{A_{\alpha}\right\}\right):\right.$ $\left\{A_{\alpha}\right\}$ is a $K$-chain in $\left.X\right\}=l(X)$.

(iv) Let $X$ be a compact space, $Y$ be a $T_{1}$ space and $f$ be a closed continuous map from $X$ onto $Y$. We show $l(Y) \leq l(X)$.

As in (iii), we start with a $K$-chain $\left\{B_{\alpha}\right\}$ on $Y$. A $K$-chain $\left\{A_{\alpha}\right\}$ in $X$ is defined as follows: $A_{0}=f^{-1}\left(B_{0}\right)$

$$
A_{\alpha}= \begin{cases}U A_{\beta} & \text { if } \alpha \text { is a limit ordinal } \\ A_{\beta} U\left(f^{-1}\left(B_{\alpha} \backslash B_{\beta}\right) \cap\left(\underset{\gamma<\beta}{\cap} \overline{A_{\beta} \backslash A_{\gamma}}\right)\right) & \text { if } \alpha=\beta+1\end{cases}
$$


CLAIM: $\left\{A_{\alpha}\right\}$ is a $K$-chain and $l\left(\left\{A_{\alpha}\right\}\right)=l\left(\left\{B_{\alpha}\right\}\right)$.

Obviously $A_{\alpha+1} \backslash A_{\alpha}$ is contained in $\overline{A_{\alpha} \backslash A_{\gamma}}$ for all $\gamma<\alpha$ by definition. Hence, it is enough to show that $A_{\alpha+1} \backslash A_{\alpha}$ is nonempty for all $\alpha$ such that $B_{\alpha+1} \backslash B_{\alpha}$ is nonempty. We prove this using (transfinite) induction. But $f\left(A_{\alpha+1} \backslash A_{\alpha}\right)$ is contained in $B_{\alpha+1} \backslash B_{\alpha}$ by definition. So, it is enough to show that $B_{\alpha+1} \backslash B_{\alpha}$ is contained in $f\left(A_{\alpha+1} \backslash A_{\alpha}\right)$. Suppose $\alpha=0$ and $y$ belongs to $B_{1} \backslash B_{0} \subset \bar{B}_{0}=\overline{f\left(A_{0}\right)} \subset f\left(\overline{A_{0}}\right)$ because $f$ is closed). Then $f^{-1}(y) \cap \bar{A}_{0}$ is nonempty. Hence, there exists a point $x$ in $\bar{A}_{0} \cap f^{-1}\left(B_{1} \backslash B_{0}\right)$ such that $f(x)=y$. Now, assume that $f\left(A_{\beta} \backslash A_{\gamma}\right)$ contains $B_{\beta} \backslash B_{\gamma}$ for all $\gamma<\beta<\alpha$ (by induction).

CASE 1: $\alpha=\beta+1$ ( $\alpha$ is not a limit ordinal).

Suppose $y$ belongs to $B_{\alpha+1} \backslash B_{\alpha}$. Since $B_{\alpha+1} \backslash B_{\alpha}$ is contained in $\bigcap_{\gamma<\alpha}\left(\overline{B_{\alpha} \backslash B_{\gamma}}\right) \subset \underset{\gamma<\alpha}{\cap} \overline{\left(A_{\alpha} \backslash A_{\gamma}\right)}$ and hence is contained in $\underset{\gamma<\alpha}{\cap}\left(\overline{A_{\alpha} \backslash A_{\gamma}}\right)$, there exists a point $x$ in $f^{-1}\left(B_{\alpha} \backslash B_{\beta}\right) \cap\left(\bigcap_{\gamma<\alpha}\left(A_{\alpha} \backslash A_{\gamma}\right)\right)$ such that $f(x)=y$.

CASE 2: $\alpha$ is a limit ordinal.

Here, $y$ is in $B_{\alpha+1} \backslash B_{\alpha}$ and hence in $B_{\alpha} \backslash B_{\beta}$ for all $\beta<\alpha$. So $y$ belongs to $\underset{\beta<\alpha}{\cap} \overline{B_{\alpha} \backslash B_{\beta}} \subset$ $\left.\underset{\beta<\alpha}{\cap} \overline{f\left(A_{\alpha} \backslash A_{\beta}\right)} \subset \underset{\beta<\alpha}{\cap} \overline{f\left(A_{\alpha} \backslash A_{\beta}\right.}\right)=f\left(\underset{\beta<\alpha}{\cap \overline{A_{\alpha} \backslash A_{\beta}}}\right)$ (because $\overline{A_{\alpha} \backslash A_{\beta}}$ is a decreasing chain). Since $X$ is compact and $\overline{A_{\alpha} \backslash A_{\beta}}$ is nonempty for all $\beta<\alpha$, there exists a point $x$ in $\cap \overline{A_{\alpha} \backslash A_{\beta}}$ such that $f(x)=y$. Since $\alpha$ is a limit ordinal, $f\left(A_{\alpha+1} \backslash A_{\alpha}\right)$ contains $B_{\alpha+1} \backslash A_{\alpha}$ follows from the induction hypothesis and the definition of $A_{\alpha}$. Thus, (iv) is proved.

Our next aim is to show that $l(X)$ is a natural extension of the derived length. For this purpose we define a new ordinal invariant $l^{\prime}(X)$ on topological spaces. $l^{\prime}(X)$ is defined as $\sup \{\alpha: \alpha$ is the derived length of a finer dispersed topology on $X\}$. We now prove

THEOREM 1.2: For any topological space $X$, it is true that $l^{\prime}(X)=1+l(X)$.

PROOF: Suppose $\tau$ is a finer dispersed topology on $X$ with derived length $d(\tau)$. Then, one can prove that $\left\{X \backslash X^{\alpha}: 0<\alpha \leq d(\tau), X^{\alpha}\right.$ is defined with respect to $\left.\tau\right\}$ is a $K$-chain with the set of all isolated points of $\tau$ as the base set, and ' $1+$ the length of this chain' is equal to $d(\tau)$. Also any $K$-chain in a finer topology is a $K$-chain in the original topology and hence we conclude that $l^{\prime}(X) \leq 1+l(X)$.

For the reverse inequality, we consider a $K$-chain $\left\{A_{\alpha}\right\}$ in $X$. Our aim is to define a finer dispersed topology on $X$ such that the derived length of this topology coincides with $\left.l\left(A_{\alpha}\right\}\right)$. For this, we define for each $\boldsymbol{x}$ in $X$ a new neighborhood system as follows. If $\boldsymbol{x}$ is a point in $A_{\alpha+1} \backslash A_{\alpha}$ for some ordinal number $\alpha$ (that has to be unique), then $\left\{\left(V_{x} \cap A_{\alpha}\right) \cup\{x\}: V_{x}\right.$ is a neighborhood of $x$ in the original topology $\}$ is declared as the new neighborhood system at $x$, and $x$ is isolated otherwise. One can verify that this defines a finer topology on $X$ whose open sets can be described as follows: 'A subset $V$ of $X$ is open if and only if for each ordinal number $\alpha$ and for each point $x$ in $V \cap\left(A_{\alpha+1} \backslash A_{\alpha}\right)$, it is true that there is a neighborhood $V_{x}$ of $x$ in the original topology such that $V_{x} \cap A_{\alpha}$ is contained in $V$.' Equivalently, this topology is generated by the family $\left\{V \supset X\right.$ : either $V$ is open in the original topology or $V=A_{\alpha} \cup\{x\}$ for some point $x$ in $A_{\alpha+1} \backslash A_{\alpha}$ and for some ordinal number $\alpha$, or $V$ is contained in $A_{0}$ or $V$ is disjoint from $\left.\cup_{\alpha} A_{\alpha}\right\}$. One can now prove that $X^{0}=X$ and for each ordinal number $\alpha \geq 1, X^{\alpha}$ (in the new topology) $=\left(\cup_{\beta} A_{\beta}\right) \backslash\left(\cup_{\beta<\alpha} A_{\beta}\right)$. It follows that this new topology is dispersed and has derived length equal to ' $1+$ the length of the $K$-chain $\left\{A_{\alpha}\right\}$ '. Thus, $l^{\prime}(X) \leq 1+l(X)$. Hence, the proof of the theorem is completed.

COROLLARY 1.3: For a dispersed space $X, d(X)=1+l(X)=l^{\prime}(X)$.

PROOF: This follows immediately from (ii) of theorem 0.3. 
REMARKS: 1 . The above corollary 1.3 justifies the title of the article. The fact that the definition of the length $l$ of a space does not involve dispersedness at all whereas $l^{\prime}$ is completely dependent on the derived lengths of finer dispersed topologies, is quite interesting $\left(\therefore l^{\prime}=1+l\right)$. The above corollary that $d(X)=1+l(X)$ for a dispersed space $X$ can be proved directly also.

2. If we observe carefully theorem 1.2 , we can easily see that $l$ is uniquely determined among all ordinal invariants in topology by the following three properties.

(i) $l$ is smaller for finer topologies.

(ii) $l$ coincides with derived length for dispersed spaces.

(iii) $l$ is minimal with respect to (i) and (ii).

Also, as an extension of $d(X)$, which is our main interest in this article, $l$ is uniquely determined by (i) and (iii).

\section{2. $d(X)$ IN TERMS OF ORDER OF A MAP.}

In his memoir [1], Kannan, defined an ordinal invariant $\delta$ for all topological spaces. The problem of characterizing $\delta$ intrinsically is left open there [F.4]. We solve this problem here in this section. In fact, the length of a space we defined above happens to be in intrinsic characterization of $\delta$. We first recall the definition of $\delta$ given in [1].

DEFINITION 2.1: Let $X$ be an arbitrary topological space chosen and fixed. Let $Y$ be any other topological space and $f: Y \rightarrow X$ be a continuous function. For any subset $A$ and $X$, we let $A_{f}^{0}=A$ and $A_{f}^{1}=f\left(\overline{\left.f^{-1}(A)\right)}\right.$. As induction hypothesis, we assume that $A_{f}^{\beta}$ is defined for all $\beta<\alpha$. Now, we let $A_{f}^{\alpha}=\left(A_{f}^{\beta}\right)_{f}^{1}$ if $\alpha=\beta+1$, a nonlimit ordinal number, and $A_{f}^{\alpha}=\cup_{\beta<\alpha} A_{f}^{\beta}$ if $\alpha$ is a limit ordinal.

Thus, $A_{f}^{\alpha}$ is defined for every ordinal $\alpha$. By a standard argument on the cardinality of $X$, one can verify that there exists an ordinal number $\alpha$ such that $A_{f}^{\alpha}=A_{f}^{\alpha+1}=\ldots$. Now we define $\sigma(A, f)=\inf \left\{\alpha: A_{f}^{\alpha}=A_{f}^{\alpha+1}\right\}$. The order of the map $f$ is defined as $\sup \{\sigma(A, f): A$ is a subset of $X\}$ and is denoted by $\sigma(f)$. Finally, $\delta(X)$ is defined as $\sup \{\sigma(f): f: Y \rightarrow X$ is continuous, $Y$ is any topological space $\}$. The existence of $\delta(X)$ as well as $\sigma(f)$ can again be established using cardinality arguments. One can check that $\delta$ is also a topological invariant. The following theorem solves the problem of Kannan.

THEOREM 2.2: For any topological space $X, l(X)=\delta(X)$.

PROOF. First we prove that $l(X) \geq \delta(X)$. For, suppose $f: Y \rightarrow X$ is a continuous map from an arbitrary topological space $Y$ and $a$ is a subset of $X$.

CLAIM: $\left\{A_{f}^{\alpha}\right\}$ is a $K$-chain and $l\left(\left\{A_{f}^{\alpha}\right\}\right)=\sigma(A, f)$.

Throughout the proof of this claim we denote $A_{f}^{\alpha}$ as $A_{\alpha}$ for notational convenience. We have to prove that for any ordinal number $\alpha, A_{\alpha+1} \backslash A_{\alpha}$ is contained in $\overline{A_{\alpha} \backslash A_{\beta}}$ for all $\beta<\alpha$. We use (transfinite) induction on $\alpha$ to prove this. Since $A_{1} \backslash A_{0}$ is contained in $\overline{A_{0}}$ can be verified without difficulty, the case $\alpha=0$ follows immediately. Now, we assume that $A_{\beta+1} \backslash A_{\beta}$ is contained in $\overline{A_{\beta} \backslash A_{\gamma}}$ for all $\gamma<\beta<\alpha$. To show that $A_{\alpha+1} \backslash A_{\alpha}$ is contained in $\overline{A_{\alpha} \backslash A_{\beta}}$ for all $\beta<\alpha$, suppose $x$ belongs to $A_{\alpha+1} \backslash A_{\alpha}$. Then, $x$ is in $A_{\alpha+1}=f\left(\overline{f^{-1}\left(A_{\alpha}\right)}\right)$. So $f^{-1}(x) \cap-\overline{f^{-1}\left(A_{\alpha}\right)}$ is nonempty. Hence there exists a point $y$ in $f^{-1}(x) \cap \overline{f^{-1}\left(A_{\alpha}\right)}$. Therefore every neighborhood of $y$ meets $f^{-1}\left(A_{\alpha}\right)$. Since $f$ is continuous, this shows that every neighborhood of $x$ meets $A_{\alpha}$. 
CASE 1: $\alpha=\beta+1$ for some ordinal number $\beta$.

In this case, it is enough to show that $x$ belongs to $\overline{A_{\mathbf{\alpha}} \backslash A_{\boldsymbol{\beta}}}$. Suppose we assume that there exists a neighborhood $U_{x}$ of $x$ such that $U_{x} \cap\left(A_{\alpha} \backslash A_{\beta}\right)$ is empty. Then, $f^{-1}\left(U_{x}\right)$ is a neighborhood of $y$ such that $f^{-1}\left(U_{x}\right) \cap f^{-1}\left(A_{\alpha}\right)$ is contained in $f^{-1}\left(A_{\beta}\right)$. But $x$ is not in $A_{\alpha}=f\left(\overline{f^{-1}\left(A_{\beta}\right)}\right)$ and hence $f^{-1}(x) \cap \overline{f^{-1}\left(A_{\beta}\right)}$ is empty. Therefore there exists a neighborhood $V_{y}$ of $y$ such that $V_{y} \cap f^{-1}\left(A_{\beta}\right)$ is empty. Hence, $V_{y} \cap f^{-1}\left(U_{x}\right)$ does not meet $f^{-1}\left(A_{\alpha} \backslash A_{\beta}\right) \cup f^{-1}\left(A_{\beta}\right)=f^{-1}\left(A_{\alpha}\right)$. This is a contradiction to the fact that $y$ is in $\overline{f^{-1}\left(A_{\alpha}\right)}$.

CASE 2: $\alpha$ is a limit ordinal number.

Here, $A_{\alpha}=\cup_{\beta<\alpha} A_{\beta}$. In this case, $x$ is not in $A_{\alpha}=\underset{\beta<\alpha}{\cup} A_{\alpha+1}=\bigcup_{\beta<\alpha} f\left(\overline{f^{-1}\left(A_{\beta}\right)}\right)$ implies that $x$ is not in $f\left(\overline{f^{-1}\left(A_{\beta}\right)}\right)$ for any $\beta<\alpha$. Hence, for any given ordinal number $\beta<\alpha$, there exists a neighborhood $V_{\beta}$ of $y$ such that $V_{\beta} \cap f^{-1}\left(A_{\beta}\right)$ is empty. Now, if $x$ does not belong to $\overline{A_{\alpha} \backslash A_{\beta}}$ for some $\beta<\alpha$, then there exists a neighborhood $U_{\beta}$ of $x$ such that $U_{\beta} \cap\left(A_{\alpha} \backslash A_{\beta}\right)$ is empty and hence $f^{-1}\left(U_{\beta}\right)$ does not meet $f^{-1}\left(A_{\alpha} \backslash A_{\beta}\right)$. So the neighborhood $f^{-1}\left(U_{\beta}\right) \cap V_{\beta}$ of $y$ does not meet $f^{-1}\left(A_{\alpha}\right)$ (as earlier). This again is a contradiction. Hence, the claim is proved. Thus, we have $\sigma(A, f)=l\left(\left\{A_{\alpha}\right\}\right)=l\left(\left\{A_{f}^{\alpha}\right\}\right)$ and hence the inequality $l(X) \geq \delta(X)$.

For the reverse inequality $l(X) \leq \delta(X)$, we consider an arbitrary $K$-chain $\left\{A_{\alpha}\right\}$ in $X$ with base set $A$. We construct a topological space $Y$ and a continuous function $f: Y \rightarrow X$ such that $\sigma(f) \geq l\left(\left\{A_{\alpha}\right\}\right)$. For each ordinal number $\alpha$, let $Y_{\alpha-1}=A_{\alpha+2} \backslash A_{\alpha}$ with relative topology from $X$. For each limit ordinal $\alpha$, let $Y_{\alpha}=A_{\alpha+1}$ with the topology generated by $\left\{V: V\right.$ is a subset of $A_{\alpha+1}$, either $V$ is an open subset of $A_{\alpha}$ or $V=\left(A_{\alpha} \backslash A_{\beta}\right) \cup\{x\}$ for some $x$ in $A_{\alpha+1} \backslash A_{\alpha}$ and some $\left.\beta<\alpha\right\}$. Finally, $Y$ is the disjoint topological sum of all these $Y_{\alpha}$ 's. A map (canonical) in $Y_{\alpha}$ for some $\alpha$. So $y$ is in $A_{\alpha+1} \backslash A_{\alpha}$ for a unique $\alpha$. Define this point in $X$ as the image of $y$ under $f$. It is easy to verify that $f$ is continuous and $\sigma\left(A_{0}, f\right)=l\left(\left\{A_{\alpha}\right\}\right)$. This proves the reverse inequality $\sigma(X) \geq l(X)$. Thus, the proof of the theorem is complete.

REMARK. One can prove more results on the length of a space such as perfect maps decrease the length, etc. The reader should consult [1] for more details. One natural question is that "which properties of the derived length of dispersed topological spaces remain true for the length of general topological spaces?" A map is called finite-to-one if the inverse image of every point is a finite subsets of the domain. It can be proved that if $X$ is a compact Hausdorff dispersed space and $f$ is a finite-to-one continuous map from $X$ onto $Y$ where $Y$ is a Hausdorff space, then $d(X)=d(Y)$. But we do not know whether this is true for the length of arbitrary compact Hausdorff spaces. Let $X$ and $Y$ be arbitrary compact Hausdorff spaces such that $Y$ is a continuous image of $X$. Then using theorem 1.1 (iv), one can prove that $l(Y) \leq l(X)$. It is interesting to know whether $l(X) \leq l(Y)$ or not when $Y$ is a finite-to-one continuous image of $X$.

ACKNOWLEDGEMENT. The author expresses his deep gratitude to Professor V. Kannan for his helpful suggestions during the preparation of this paper. The author likes to thank the referee for suggesting the present title and for pointing out an error in the earlier version of the article.

Financially supported by Council of Scientific and Industrial Research, India.

Current Address: Department of Mathematics, Goa University, Bambolim, Santa Cruz P.O., Goa, India 403005.

\section{REFERENCES}

[1] KANNAN, V., Ordinal invariants in topology, Memoirs, Amer. Maths. Soc., Number 245 (1981).

[2] SEMADENI, Z., Sur les ensembles clairsemes, Rozprrrawy Mat. 19 (1959).

[3] KANNAN, V. and RAJAGOPALAN, M., Scattered spaces II, III. J. Math., 21 (1977) 735-751.

[4] LACEY, E. H., Isomeric Theory of Classical Banach Spaces, Springer-Verlag (1974). 


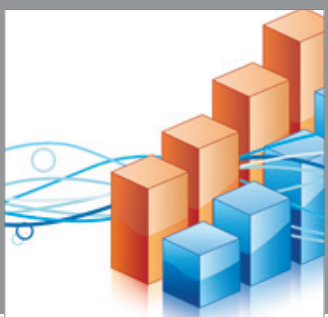

Advances in

Operations Research

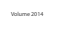

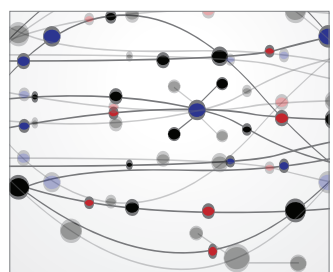

\section{The Scientific} World Journal
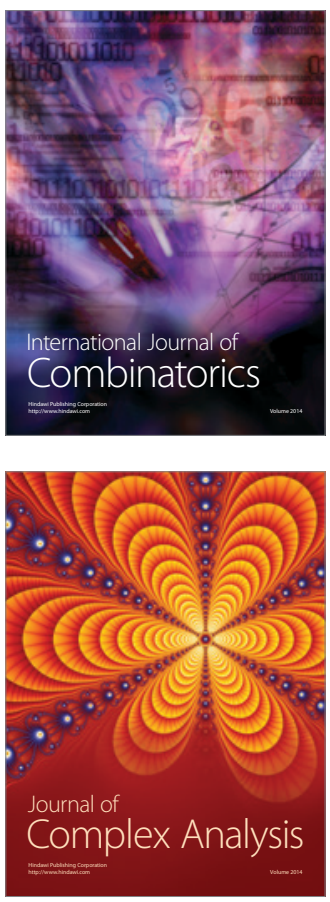

International Journal of

Mathematics and

Mathematical

Sciences
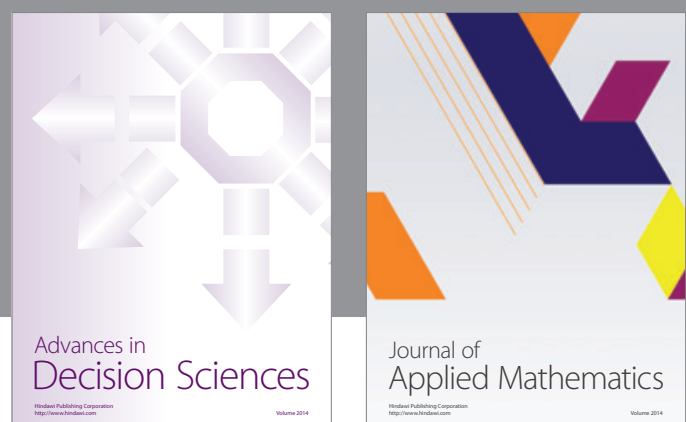

Journal of

Applied Mathematics
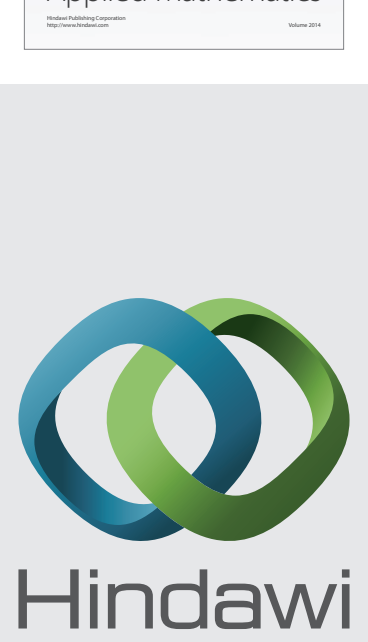

Submit your manuscripts at http://www.hindawi.com
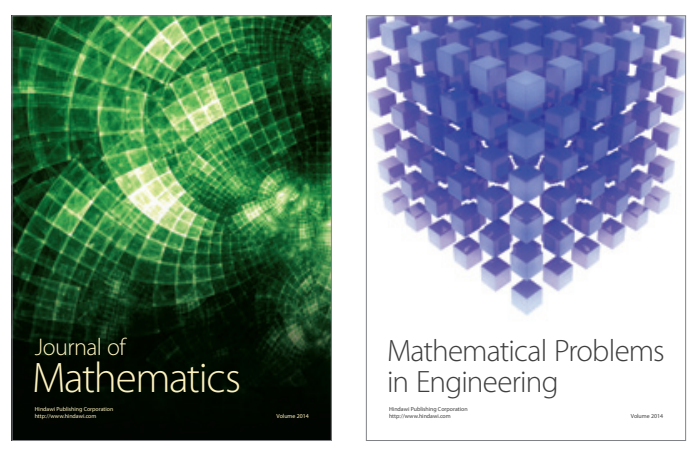

Mathematical Problems in Engineering
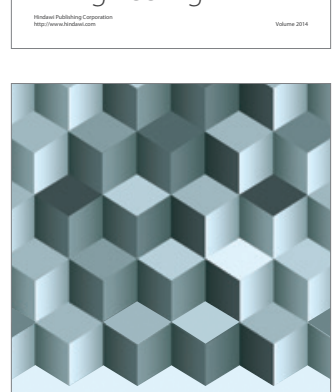

Journal of

Function Spaces
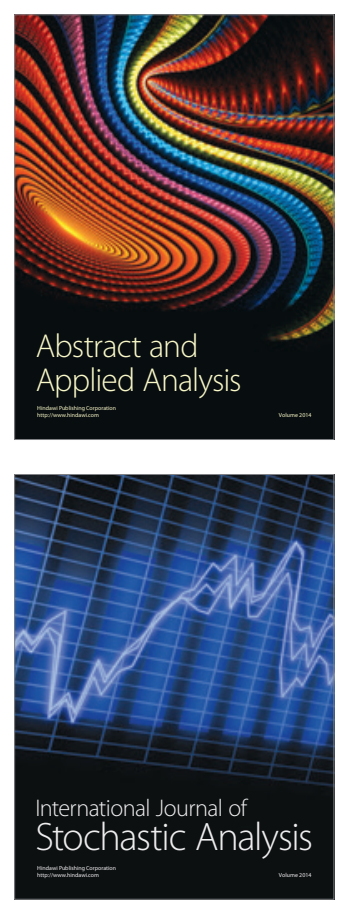

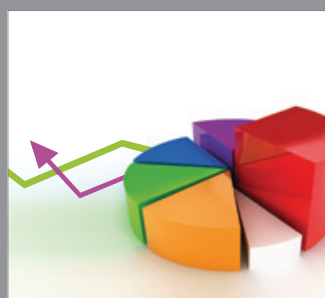

ournal of

Probability and Statistics

Promensencen
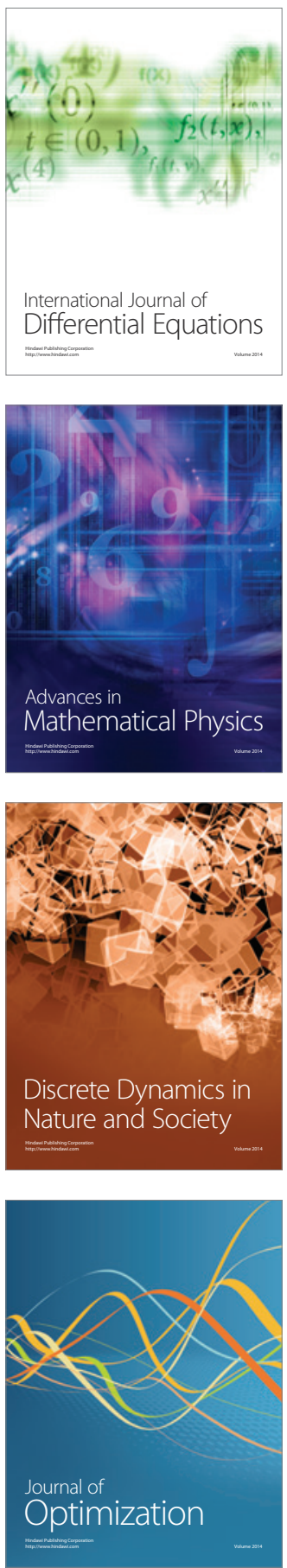